(59.14 vs. $46.87, \mathrm{P}=0.000$ ), more number of people suffering from coronary heart disease ( 7 vs. $5, P=0.016)$. The glomerular filtration rate was lower (58.5 vs. $71.6, P=0.000$ ), with lower urinary creatinine, uric acid, and urinary potassium. Conclusion: Gout patients with and without simple renal cysts have significant differences in age, hypertension, cardiovascular disease, GFR, serum creatinine, urine creatinine, and urine potassium.

REFERENCES:

[1]N. Dalbeth, T.R. Merriman, L.K. Stamp, Gout, Lancet 388(10055) (2016) 2039-2052.

[2] E.M. Hasegawa, R. Fuller, M.C. Chammas, F.M. de Mello, C. Goldenstein-Schainberg, Increased prevalence of simple renal cysts in patients with gout, Rheumatol Int 33(2) (2013) 413-6.

[3] Y. Han, M. Zhang, J. Lu, L. Zhang, J. Han, F. Zhao, H. Chen, Y. Bao, W. Jia, Hyperuricemia and overexcretion of uric acid increase the risk of simple renal cysts in type 2 diabetes, Sci Rep 7(1) (2017) 3802

Table 1. Clinical characteristics of gout patients

\begin{tabular}{llll}
\hline & Renal cyst $(\mathrm{n}=56)$ & Without Renal cyst $(\mathrm{n}=144)$ & $\mathrm{P}$ \\
\hline Disease duration, (month) & $98.7( \pm 64.1)$ & $91.2( \pm 67.0)$ & 0.468 \\
Age, (year) & $59.14( \pm 14.3)$ & $46.78( \pm 15.9)$ & 0.000 \\
Gender, $\mathrm{n}(\mathrm{F} / \mathrm{M})$ & $7 / 49$ & $11 / 133$ & 0.281 \\
Smoking history, $\mathrm{n}(\%)$ & $18(32.1 \%)$ & $47(32.6 \%)$ & 0.946 \\
Drinking history, $\mathrm{n}(\%)$ & $10(17.9 \%)$ & $32(22.2 \%)$ & 0.496 \\
Hypertension, $\mathrm{n}(\%)$ & $31(55.3 \%)$ & $49(34.0 \%)$ & 0.006 \\
Diabetes, $\mathrm{n}(\%)$ & $9(16.1 \%)$ & $15(10.4 \%)$ & 0.269 \\
CVDs, $\mathrm{n}(\%)$ & $7(12.5 \%)$ & $5(3.4 \%)$ & 0.016 \\
Nephrolithiasis, $\mathrm{n}(\%)$ & $14(25 \%)$ & $43(29.9 \%)$ & 0.494 \\
UA, $(\mu \mathrm{mol} / \mathrm{L})$ & $494.8( \pm 158.0)$ & $544.3( \pm 121.0)$ & 0.037 \\
Serum creatinine, $(\mu \mathrm{mol} / \mathrm{L})$ & $139.4( \pm 57.2)$ & $116.5( \pm 35.45)$ & 0.007 \\
GFR, $(\mathrm{ml} / \mathrm{L})$ & $58.5( \pm 22.5)$ & $71.6( \pm 22.3)$ & 0.000 \\
FEUA, $(\%)$ & $7.0( \pm 3.2)$ & $6.0( \pm 3.2)$ & 0.052 \\
Urine creatinine, $(\mu \mathrm{mol} / \mathrm{L})$ & $4687.09( \pm 1832.9)$ & $5565.2( \pm 2599.8)$ & 0.008 \\
Urine Uric acid, $(\mu \mathrm{mol} / \mathrm{L})$ & $1204.9( \pm 772.0)$ & $1542.1( \pm 1048.5)$ & 0.030 \\
Urine sodium, $(\mathrm{mmol})$ & $132.1( \pm 68.7)$ & $131.2( \pm 76.6)$ & 0.939 \\
Urine potassium, $(\mathrm{mmol})$ & $25.6( \pm 12.5)$ & $31.8( \pm 14.2)$ & 0.005 \\
\hline
\end{tabular}

Disclosure of Interests: None declared.

DOI: 10.1136/annrheumdis-2021-eular.3702

\section{AB0646 COMPARATIVE ANALYSIS OF ASYMPTOMATIC HYPERURICEMIA AND GOUT - AN IMPACT ON KIDNEY STRUCTURE AND FUNCTION}

M. Hristova ${ }^{1}$, A. Kundurdzhiev ${ }^{1}$, T. Kundurzhiev ${ }^{2}$, R. Gancheva ${ }^{3} .{ }^{1}$ University Hospital St Ivan Rilski, Medical University Sofia, Internal Medicine, Sofia, Bulgaria; ${ }^{2}$ Faculty of Public Health, Medical University Sofia, Department of Occupational Medicine, Sofia, Bulgaria; ${ }^{3}$ University Hospital St Ivan Rilski, Medical University Sofia, Rheumatology, Sofia, Bulgaria

Background: The significance of asymptomatic hyperuricemia $(\mathrm{AH})$ continues to be debated. At the population level, asymptomatic hyperuricemia is associated with multiple comorbidities, including hypertension, coronary artery disease, diabetes and chronic kidney disease.

Objectives: To investigate the impact of asymptomatic hyperuricemia on renal functional and structural parameters in comparison with symptomatic gout. Methods: The subjects included in the study were divided into two groups - 46 patients with asymptomatic hyperuricemia and 18 diagnosed with gout according to ACR/ EULAR 2015 criteria. $^{2}$ For renal function assessment urine analysis, albuminuria, estimated glomerular filtration rate (eGFR) based on Chronic Kidney Disease Epidemiology Collaboration (CKD-EPI) equation were used. Diagnostic ultrasound as well as renal biopsy were performed to evaluate structural and histological kidney changes. Statistical analysis was performed using SPSS 22.0 software (SPSS Inc, Chicago, USA). Results: There were no significant differences between the two groups on serum uric acid levels, albuminuria, and eGFR. However, erythrocyturia $(p=0.047)$ and nephrolithiasis $(p<0.001)$ prevailed significantly in patients with gout, $66.7 \%$ and $61.1 \%$ respectively. We did not find any histological differences between the two groups regarding the number of affected glomeruli, tubular atrophy and percentage of interstitial fibrosis based on light microscopy scans.

Conclusion: Our results indicate that patients with $\mathrm{AH}$ need renal screening since both $\mathrm{AH}$ and gout show similar kidney changes. Further research is needed to elucidate the role of early treatment with xanthine oxidase inhibitor for asymptomatic hyperuricemia as prevention of complications such as erythrocyturia and nephrolithiasis. REFERENCES:

[1] Yip K, Cohen RE, Pillinger MH. Asymptomatic hyperuricemia: is it really asymptomatic? CurrOpin Rheumatology 2020; 32(1):71-79 doi: 10.1097/ BOR.0000000000000679.

[2] Neogi T, Jansen TL, Dalbeth N et al. 2015 Gout classification criteria: an American College of Rheumatology/European League Against Rheumatism collaborative initiative. Arthritis Rheumatogy 2015;67(10):2557-68. doi: 10.1002/art.39254
Disclosure of Interests: None declared.

DOI: 10.1136/annrheumdis-2021-eular.4306

\section{Infection-related rheumatic diseases}

\section{AB0647 \\ CATASTROPHIC ANTIPHOSPHOLIPID SYNDROME TRIGGERED BY CONCURRENT VZV AND HSV INFECTION. A CASE REPORT}

L. Kakoullis ${ }^{1}$, K. Parperis ${ }^{2,3}$, S. Psarelis ${ }^{4}$, E. Papachristodoulou' ${ }^{1}$, G. Panos ${ }^{5,6}$ ${ }^{1}$ University of Cyprus Medical School, Department of Medicine, Nicosia, Cyprus; ${ }^{2}$ University of Cyprus Medical School, Department of Medicine, Division of Rheumatology, Nicosia, Cyprus; ${ }^{3}$ University of Arizona College of Medicine, Department of Medicine, Division of Rheumatology, Phoenix, Arizona, United States of America; ${ }^{4}$ Nicosia General Hospital, Department of Rheumatology, Nicosia, Cyprus; ${ }^{5}$ University General Hospital of Patras, Department of Internal Medicine, Section of Infectious Diseases, Patras, Greece; ${ }^{6}$ University of Cyprus Medical School, Department of Internal Medicine, Nicosia, Cyprus

Background: Catastrophic antiphospholipid syndrome (CAPS) is a severe autoimmune condition, characterized by multiorgan failure due to thromboses and/or hemorrhage. Concurrent HSV/VZV infection is an exceedingly rare event, occurring mostly in immunocompromised patients. Herein, we report the first case of CAPS triggered by concurrent HSV/VZV infection, manifesting with zosteriform rash and haemorrhages at multiple sites.

Objectives: To describe a case of CAPS provoked by concurrent HSV/VZV infection, manifesting with zosteriform rash and haemorrhages at multiple sites. Methods: A 36-year-old male, with a history of antiphospholipid syndrome (APS) presented with a 5-day history of epigastric pain, associated with fever, hematuria, rash and swelling in bilateral ears. Past medical history was remarkable for APS, based on 4 episodes of DVT/PE and triple positive antiphospholipid antibodies, on chronic well-maintained warfarin anticoagulation. On examination, a purpuric rash was evident on the right side of the neck as well as on both ears, consistent with a C3 dermatomal distribution (Figure 1). Laboratory studies revealed anemia, thrombocytopenia and acute kidney injury.

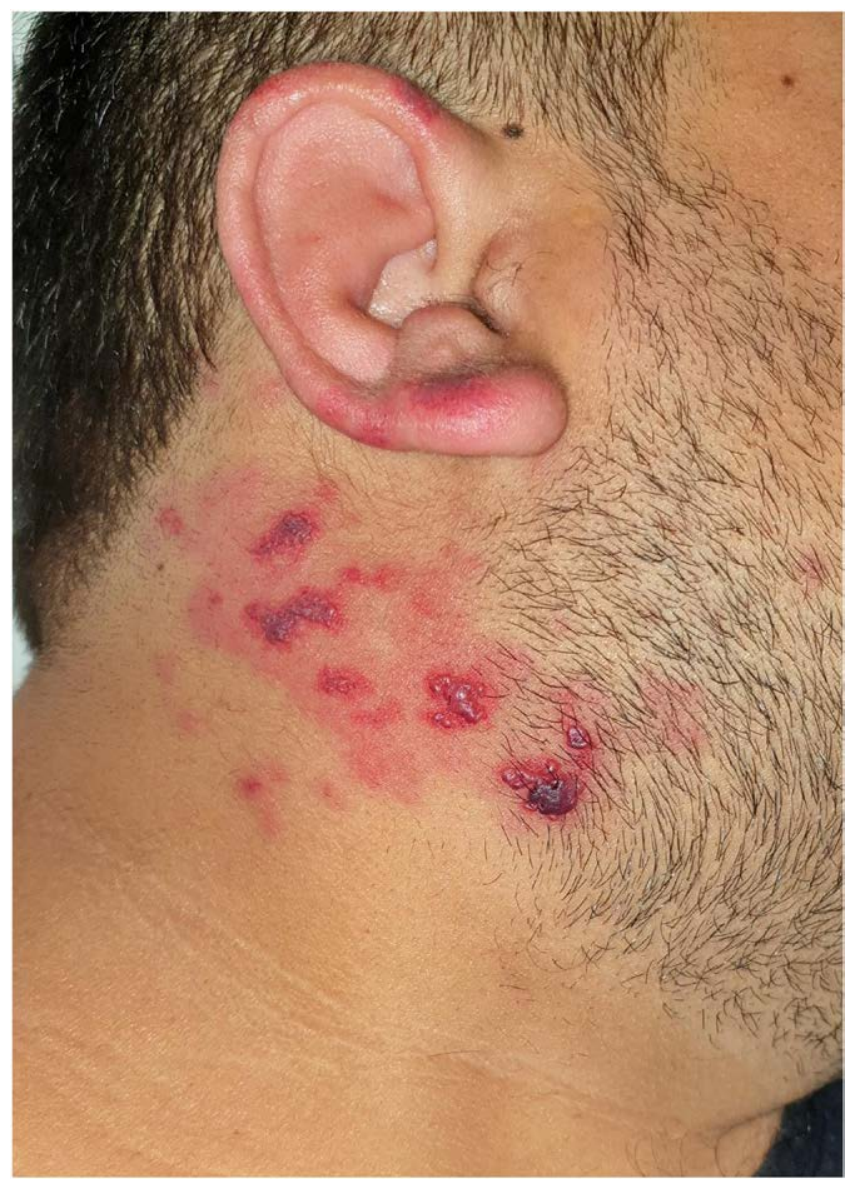

Figure 1. 